\title{
Parametric Generation of Middle and Far Infrared Radiation in GaAs-Based Semiconductor Lasers and Waveguides
}

\author{
V.Ya. Aleshikin ${ }^{a}$, A.A. Afonenko ${ }^{b}$, A. Biryukov $^{c}$, \\ V.I. Gavrilenko ${ }^{a}$, A.A. Dubinov ${ }^{a}$, Vl.V. Kocharovsky $^{d}$, \\ S.V. Morozov ${ }^{a}$, K.V. Maremynin ${ }^{a}$, S.M. NeKorkin ${ }^{c}$, \\ B.N. ZVONKOV ${ }^{c}$ AND N.B. ZVONKOV ${ }^{c}$ \\ ${ }^{a}$ Institute for Physics of Microstructures RAS \\ GSP-105, Nizhny Novgorod, 603950, Russia \\ ${ }^{b}$ Byelorussian State University, 220050, Minsk, Byelorussia \\ ${ }^{c}$ Physical-Technical Institute of Nizhny Novgorod State University \\ 23, Gagarina av., Nizhny Novgorod, 603950, Russia \\ ${ }^{d}$ Institute of Applied Physics of RAS \\ 46, Ulyanova Str., Nizhny Novgorod, 603950, Russia
}

A possibility of parametric generation of middle and far infrared radiation due to the lattice nonlinearity in semiconductor laser diode and waveguide is discussed. Three possible waveguide constructions for effective generation are considered. The parametric generation method is shown to provide generation power of the order of milliwatt for middle infrared radiation and of tens of microwatt for far infrared radiation using modern quantum well laser diodes.

PACS numbers: 42.55.Px, 42.65.-k

\section{Introduction}

Weak green light is usually seen on the mirrors of the GaAs-based laser diodes emitting near $1 \mu \mathrm{m}$ wavelength radiation. This green light is generated due to the optical nonlinearity of GaAs. Though the absorption coefficient of green light is over $10^{5} \mathrm{~cm}^{-1}$ and the observed green radiation is generated in a less than one micron thick region near the mirror, this green light is visible with a naked eye. It is an effective demonstration of high optical nonlinearity of GaAs.

It would be interesting to analyze a possibility of the mid and far infrared radiation generation in a laser diode simultaneously emitting in two spectral lines in 
the near infrared region, or in the external waveguide pumped by two laser diodes. It should be noted that quantum well laser diodes are able to generate simultaneously at two different frequencies [1]. In this paper a possibility of difference frequency generation in laser diodes and waveguides based on GaAs is discussed.

\section{Lattice nonlinearity in GaAs}

In $\mathrm{A}_{3} \mathrm{~B}_{5}$ semiconductors with zinc-blende structure the second-order optical nonlinearity can be observed. Due to this nonlinearity two propagating waves with frequencies $\omega_{1}$ and $\omega_{2}$ induce the polarization waves at double, sum, and difference frequencies. The electrical induction at difference frequency can be represented in the form

$$
D_{i}\left(\omega_{1}-\omega_{2}\right)=\varepsilon\left(\omega_{1}-\omega_{2}\right) E\left(\omega_{1}-\omega_{2}\right)+\varepsilon_{i j k}^{(2)}\left(\omega_{1}, \omega_{2}\right) E_{j}\left(\omega_{1}\right) E_{k}\left(\omega_{2}\right),
$$

here $\varepsilon\left(\omega_{1}-\omega_{2}\right)$ is the ordinary dielectric constant for difference frequency, $\varepsilon_{i j k}^{(2)}\left(\omega_{1}, \omega_{2}\right)$ is the second-order optical nonlinear tensor. The origin of the second-order optical nonlinearity in GaAs is the anharmonicity of the transverse optical lattice vibrations induced by electromagnetic radiation. This is the reason why this nonlinearity is called the lattice nonlinearity. The theory of the lattice nonlinearity for zinc-blende structure materials was developed by Flytzanis [2].

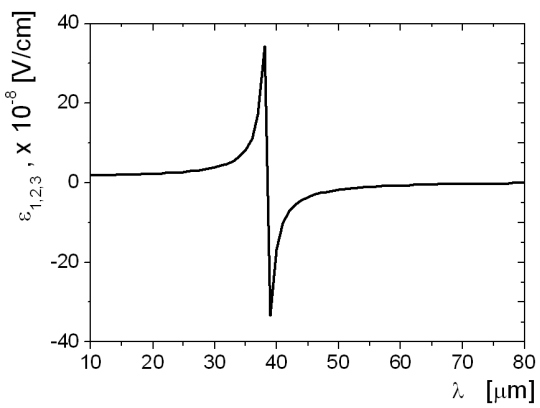

Fig. 1. Dependence of tensor $\varepsilon_{123}^{(2)}$ on difference mode wavelength in GaAs. $\lambda_{2}=1 \mu \mathrm{m}$ is fixed.

In the mentioned materials all the elements of the tensor $\varepsilon_{i j k}^{(2)}$ with different $i, j, k$ are equal to each other, while all other elements equal zero. The dependence of $\varepsilon_{123}^{(2)}\left(\omega_{1}, \omega_{2}\right)$ on the difference harmonic wavelength $\lambda$ is presented in Fig. 1 $\left(\lambda_{2}=1 \mu \mathrm{m}\right.$ is fixed). From Fig. 1 one can see that there is a resonance at the wavelength corresponding to the transverse optical phonon energy. For the shorter wavelengths the nonlinear coefficient leads to saturation $\varepsilon_{i j k}^{(2)}\left(\omega_{1}, \omega_{2}\right) \approx$ $1.7 \times 10^{-8} \mathrm{~V} / \mathrm{cm}$.

Let us note that the GaAs-based waveguides and laser structures usually are grown on (001) surface and the radiation propagates along [110] or equivalent directions. In the case when both near infrared modes are TE modes (this usually 
is observed in laser diodes), TM modes are excited at difference, sum, and double frequencies due to the lattice nonlinearity.

\section{Generation of the difference harmonic using fundamental and excited waveguide modes}

The phase velocity of the induced polarization wave at the difference frequency is $v=\left(\omega_{1}-\omega_{2}\right) /\left(k_{1}-k_{2}\right)$. When both near infrared modes are fundamental ones $\left(\mathrm{TE}_{0}\right)$ in InGaP/GaAs/InGaP or AlGaAs/GaAs/AlGaAs waveguide, calculations show that the value of an effective refractive index for polarization wave exceeds 4 in 10-100 $\mu \mathrm{m}$ wavelength region. For effective generation of the difference frequency mode to take place the phase velocities of the polarization wave and propagating mode at this frequency must coincide (phase matching condition). Let us note that the refractive index of GaAs in the normal dispersion region $(1-30 \mu \mathrm{m})$ is less than 3.6. Therefore in this region phase velocity of induced polarization wave is less than that of the waveguide mode, and it is impossible to satisfy the phase matching condition. The physical origin is the normal dispersion of the refractive index in this region.

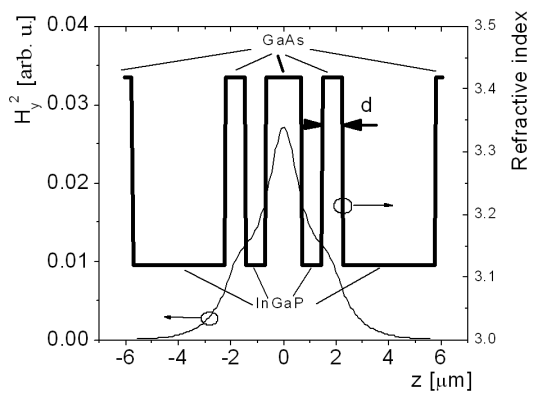

Fig. 2. Scheme of the laser waveguide design and $z$-coordinate dependences (across the structure) of the real part of the refractive index and squared magnetic field in the difference mode.

The phase matching condition can be satisfied if the highest frequency $\left(\omega_{1}\right)$ mode is an excited mode of the waveguide. Indeed, the phase velocity of excited mode is greater than that of the fundamental mode of the same frequency. This feature allows to compensate normal dispersion of the refractive index in the near infrared region and provides phase matching for the difference mode. The laser waveguide design made possible to satisfy phase matching condition at $10 \mu \mathrm{m}$ wavelength, as presented in Fig. 2. In this case $\omega_{1}$ frequency mode is $\mathrm{TE}_{2}$ and $\omega_{2}$ frequency mode is $\mathrm{TE}_{0}$. The dependence of the difference mode squared magnetic field is shown in the same plot. Detailed description of this waveguide is presented in Ref. [3]. 


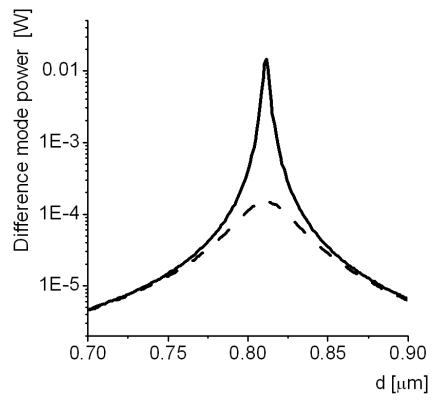

Fig. 3. The difference mode power output versus central GaAs layer thickness for two values of the imaginary part of refractive index, $n^{\prime}=10^{-4}$ (solid line) and $10^{-3}$ (dashed line).

The calculated dependence of the difference frequency power on the width of the central GaAs layer is shown in Fig. 3. The maximum of power corresponds to the phase matching. Here and further in calculation of the difference mode power we have supposed that the waveguide ridge width equals $100 \mu \mathrm{m}$ and the powers of both near infrared modes are $10 \mathrm{~W}$. The solid and dashed lines correspond to the doping levels $10^{17} \mathrm{~cm}^{-3}$ and $10^{18} \mathrm{~cm}^{-3}$, respectively. We have used the value $10^{-4}$ for imaginary part of the refractive index at the difference frequency in low doped material and the value $10^{-3}$ in the higher doped material [4]. The difference mode wavelength is $8.66 \mu \mathrm{m}$ at $\lambda_{1}=0.95 \mu \mathrm{m}$ and $\lambda_{2}=1.066 \mu \mathrm{m}$. From Fig. 3 one can see that power maximum in the difference mode is of the order of ten milliwatt.

To provide the simultaneous generation of two near infrared modes in [3] it was suggested to make use of special locations of quantum wells inside the laser waveguide. The quantum wells that generate the $\mathrm{TE}_{0}$ mode must be placed in the nodes of the $\mathrm{TE}_{2}$ mode.

The drawback of this scheme is stringent requirements for accuracy of layer widths, including quantum wells, the impossibility of tuning to the phase matching condition. In addition, such type of waveguide is unsuitable for generation of radiation in the far infrared region.

\section{Difference mode generation in surface plasmon waveguide}

Another method for nonlinear difference mode generation can be realized at frequency near the optical phonon. In this region the refractive index becomes high and it appears possible to provide the phase matching condition when both near infrared modes are $\mathrm{TE}_{0}$. In this wavelength region and in far infrared region it is advantageous to use the surface-plasmon waveguide, because it does not require thick cladding layers. However, for near infrared modes the ordinary dielectric waveguide should be used.

The $z$-coordinate dependence of the magnetic field amplitudes for waveguide discussed in Ref. [5] is presented in Fig. 4. In the considered structure the surface- 


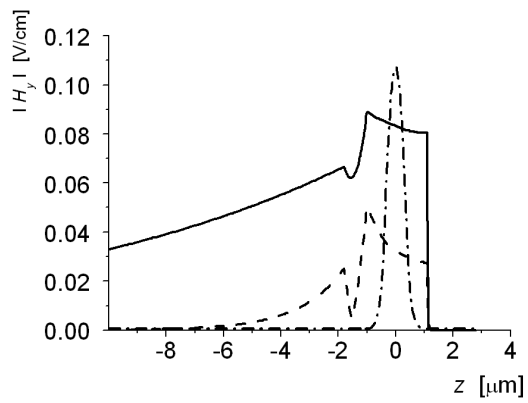

Fig. 4. Dependence of the magnetic field amplitudes on $z$-coordinate in surface-plasmon waveguide. Solid line and dashed lines correspond to the difference modes with $48 \mu \mathrm{m}$ and $29 \mu \mathrm{m}$ wavelength respectively. Dash-dotted line stands for the reduced amplitude of the near infrared ones.

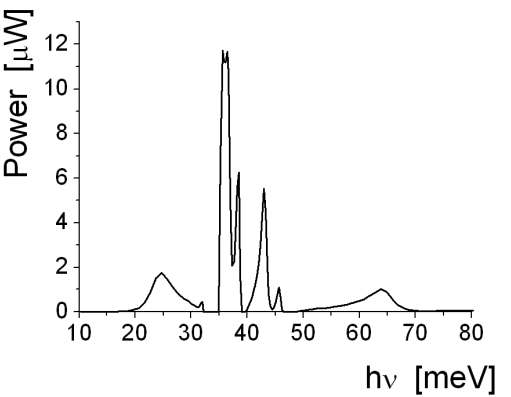

Fig. 5. Calculated dependence of difference mode power output versus photon energy for surface plasmon waveguide. $\lambda_{2}=1 \mu \mathrm{m}$.

-plasmon waveguide is formed by Au layer $(z>1 \mu \mathrm{m})$ and highly doped GaAs layer $(-2 \mu \mathrm{m}<z<-1 \mu \mathrm{m})$. Dash-dotted line shows the magnetic field amplitudes of near infrared modes. Calculated dependence of the difference mode power versus frequency for this structure is shown in Fig. 5 .

An advantage of this scheme is a weak sensitivity of the difference mode power to structure parameters [5]. A drawback is the high absorption coefficient due to the free carrier and phonon absorption. As a result the generated power is less than $10 \mu \mathrm{W}$. Let us note that due to the resonant behavior of the nonlinear optical coefficient the generated power becomes maximal near optical phonon frequency regardless of high phonon absorption.

\section{Usage of diffraction grating}

It is possible to realize an effective difference mode generation if the diffraction grating on the waveguide top is used. In this case there is no need in the waveguide for difference mode (although it is still required for near infrared modes) because the difference frequency wave is emitted through the grating as shown in Fig. 6. The directional diagram of radiation of a such structure has a pronounced 
peak. The width of the peak reduces with shortening of $\lambda$ and increase in the structure size. The effective generation is due to the phase matching condition between one of the spatial harmonics localized near the grating and the polarization wave with difference frequency. The phase velocities of spatial harmonics are governed by the grating period. Calculation shows that for the effective difference frequency generation the grating period should be of the order of $\lambda / 4$ for GaAs-based waveguide. Let us note that diffraction grating has been used for light emission from dielectric waveguides [6].

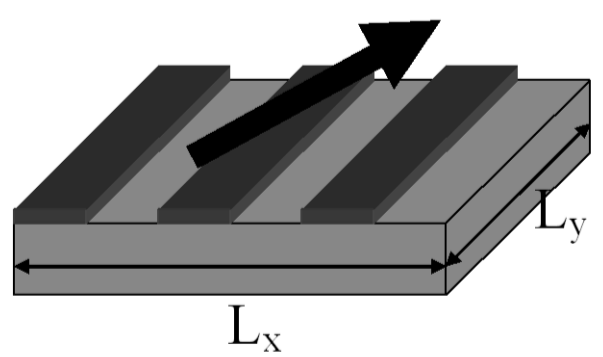

Fig. 6. Sketch of the waveguide with diffraction grating. Arrow shows the emission direction of the difference frequency wave.

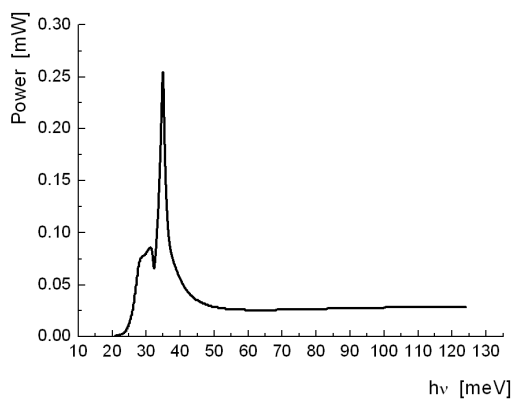

Fig. 7. Calculated dependence of difference mode power output versus photon energy for the waveguide with diffraction grating (Fig. 6). $L_{x}=1 \mathrm{~cm}, L_{y}=100 \mu \mathrm{m}$.

The results of calculation of the difference frequency power for waveguide with $1 \mathrm{~cm}$ long metal grating is presented in Fig. 7. Calculations were carried out using the method described in [6]. We assumed that both near infrared modes are $\mathrm{TE}_{0}$ modes. The power peak is predicted near the optical phonon energy and the power is almost constant for larger photon energies. The diffraction grating parameters were chosen optimal for all photon energies. As seen from Fig. 7, the discussed construction can provide the generation power of the order of $0.3 \mathrm{~mW}$ near the optical phonon frequency and about $30 \mu \mathrm{W}$ for middle infrared range. 


\section{Conclusions}

Summing the results of theoretical analysis of suggested constructions for difference mode generation in GaAs based waveguides based on lattice nonlinearity, we can conclude that the use of modern laser diodes allows to generate radiation up to $1 \mathrm{~mW}$ in $10 \mu \mathrm{m}$ wavelength region and about tens of microwatt in $40 \mu \mathrm{m}$ wavelength region at room temperature. Generation can be realized in external waveguides, or in specially constructed laser waveguides.

\section{Acknowledgments}

The work was partially supported by ISTC (grant 2293), RFBR (grant 04-0217432), Russian Academy of Sciences, Russian Ministry of Education and Science. Authors thank the European Commission project Center of Excellence in Processing, Research and Application of Advanced Materials for assistance to attend 12th International Symposium on Ultrafast Phenomena in Semiconductors.

\section{References}

[1] S. Ikeda, A. Shimizu, T. Hara, Appl. Phys. Lett. 55, 1155 (1989).

[2] C. Flytzanis, Phys. Rev. B 6, 1264 (1972).

[3] V.Ya. Aleshkin, A.A. Afonenko, N.B. Zvonkov, Semiconductors 35, 1203 (2001).

[4] Handbook of Optical Constants of Solids, Ed. E.D. Palik, Academic, Orlando, FL 1985.

[5] A.A. Afonenko, V.Ya. Aleshkin, A.A. Dubinov, Semiconductors 38, 239 (2004).

[6] V.P. Shestopalov, Riemann-Hilbert Method in the Theory of Diffraction and Propagation of Electromagnetic Waves, Kharkov University Press, Kharkov 1971. 